\title{
Dinero-capital de la Asignación Universal por Hijo para la Protección Social en un barrio marginal de la ciudad de Paraná, Argentina*
}

\author{
Capital-Money of the Program Children's Universal Allowance for Social \\ Protection in a Marginal Neighborhood of Paraná, Argentina
}

\author{
Andrés Dapuez ${ }^{* *}$ \\ Universidad Nacional de Entre Ríos (UNER) \\ Consejo Nacional de Investigaciones Científicas y Técnicas (Conicet), Argentina \\ University of Maryland at College Park \\ Observatorio de Transformaciones Socioeconómicas (ANID/PCI/Max Planck Institute), Chile
}

DOI: $10.22380 / 2539472 X .1155$

\section{RESUMEN}

En este artículo analizo el caso de una madre y su hijo, beneficiarios de la Asignación Universal por Hijo, una transferencia monetaria condicionada que exige la escolarización del menor. El abandono de la escuela y la entrada esporádica del joven en el mercado laboral desencadenan en el ámbito doméstico disputas y proyecciones económicas a corto y largo plazo sobre lo que varios autores han denominado capital humano y dinero-capital, nociones centrales en estas transferencias. En cuanto ambos beneficiarios interpretan la exigencia educativa del programa como condición necesaria para alcanzar una vida mejor, concluyo que los diseñadores de la política pública y sus destinatarios coinciden en caracterizar una forma cada vez más popular de dinero del que se esperan retornos económicos a futuro, como si se tratara de una inversión.

Palabras clave: capital humano, transferencias monetarias condicionadas, Asignación Universal por Hijo para la Protección Social, antropología del capital, dinero-capital.

\section{ABSTRACT}

In this paper I analyze the case of a mother and her child, beneficiaries of the Universal Child Allowance, a conditional cash transfer that requires the minor's schooling. School dropouts and the sporadic entry of young people into the labor market trigger domestic disputes and short-term and long-term economic projections on what various authors have called human capital and money-capital, central notions in these transfers. As both beneficiaries interpret the educational requirement of the program as a necessary condition to achieve a better life, I conclude that the designers of public policy and their recipients coincide in characterizing an increasingly popular form of money from which future economic returns are expected, as if it were an investment.

Keywords: human capital, conditional cash transfer, Children Universal Allowance for Social Protection, anthropology of capital, capital-money.

El autor agradece las lecturas críticas de los evaluadores anónimos de este artículo y sus sugerencias. El texto ha ganado claridad conceptual gracias a ellos.

. $\quad$ https://orcid.org/0000-0003-0253-9619 / afdapuez@fceco.uner.edu.ar 


\section{El capital desde uno de sus márgenes}

¿Qué ocurre cuando los beneficiarios de un programa de transferencias monetarias condicionadas, en un barrio periférico de la ciudad de Paraná1 ${ }^{1}$ se anticipan a su finalidad y reflexionan sobre las posibilidades de mejorar sus ingresos a futuro invirtiendo en educación o, en otras palabras, cuando los destinatarios de la política discuten sus posibilidades y la conveniencia de acumular lo que en el discurso experto de los economistas se denomina capital humano? Las investigaciones sobre capital humano, iniciadas en la escuela monetarista de Chicago, no solo han delimitado y definido un fenómeno, sino que también han creado un nuevo capital en el cual invertir y del cual esperar retornos. Las entrevistas realizadas con una madre y un hijo beneficiario de la Asignación Universal por Hijo (en adelante, AUH) sirvieron para indagar qué tipo de entendimiento tenían no solamente de la finalidad a largo plazo de la AUH, sino también del proceso de inversión en salud y educación del menor, en cuanto activos que le aumentarían las posibilidades de obtener mejores ingresos a futuro ${ }^{2}$. Aunque no usen las mismas palabras que los economistas, ellos interpretan el propósito de acumular capital humano que diversas políticas públicas señalan como condición necesaria, pero no suficiente, para tener una vida mejor. Así, diseñadores e implementadores de políticas públicas como las transferencias monetarias y los sectores beneficiarios de estas coinciden en caracterizar una forma cada vez más popular de dinero: un dinero-capital del que se esperan retornos económicos.

Para precisar más el análisis de las expectativas que la transferencia produce, defino como dinero-capital el efectivo de esta transferencia, considerado como un activo o asset del cual se espera un flujo de ingreso futuro. Como se verá, esta definición es mucho más simple y abarcadora que la fórmula marxista

1 Paraná es una ciudad argentina situada en la provincia de Entre Ríos, que tiene una población de 339.930 habitantes distribuidos en 80.284 hogares (Indec 2010). Según la Encuesta Permanente de Hogares, en el tercer trimestre del 2018 el 10,5\% de los hogares paranaenses recibían una ayuda monetaria proveniente del Estado u otra organización (Argentina, Ministerio de Economía, Hacienda y Finanzas, Gobierno de Entre Ríos, Dirección General de Estadísticas y Censos 2018).

2 Si bien el propósito de acumulación de capital humano está siendo discutido a la luz del desempleo o subempleo de grupos hipercalificados educacionalmente en Europa, donde algunos autores ven nacer a la nueva clase altamente educada del precariado (Standing 2011), en Argentina, sectores políticos y sociales argumentan que el acceso a educación, salud y habilidades laborales específicas son condiciones de posibilidad para salir de la pobreza. La efectividad a largo plazo de las transferencias monetarias - por ejemplo, coadyuvar a romper el ciclo de reproducción de la pobreza- parece convivir perfectamente con su efectividad a corto plazo: administrar con paliativos las expectativas de una nueva clase social mundial compuesta por más de 2.000 millones de precarizados que trabajan en la economía informal (OIT 2018). 
dinero-mercancía-dinero. En esta última, Marx (2000) considera que "la circulación de dinero como capital es un fin en sí mismo, porque la valorización del valor toma su sitio solamente dentro de este movimiento renovado. El movimiento del capital por lo tanto es sin límites” (108).

La sintaxis marxista presupone así no solamente la existencia de la mercancía como forma sobre la que se construye la moneda, sino también la preexistencia de un dinero simple; un dinero que podría escapar al movimiento sin límites del capital. Por el contrario, las preocupaciones manifestadas por las receptoras-administradoras ${ }^{3}$ de la AUH y sus hijas/os, los beneficiarios de esta política, con relación al objeto capital humano, al mandato de su acumulación y a su dinero, parecen ir en contra de cualquier noción de dinero simple. Recurro aquí a sus reflexiones y experiencias, teniendo en cuenta los objetivos del programa AUH. Según un documento del Observatorio de la Seguridad Social de la República Argentina:

La Asignación Universal por Hijo para Protección Social es uno de los programas más ambiciosos que se ha implementado en los últimos años en Argentina, no solo por sus efectos sobre la reducción de la pobreza y la indigencia en el corto plazo, sino además por ser una política de desarrollo que incentiva la inversión en capital humano y en el cuidado de la salud, con claros efectos en el largo plazo. (Anses 2011, 5)

La AUH es un programa nacional de transferencias monetarias condicionadas (TMC) cuyos beneficiarios son los infantes, niñas, niños y jóvenes de 0 a 18 años que concurran a la escuela y cumplan con el calendario estatal de vacunación y salud, así como los discapacitados de cualquier edad ${ }^{4}$. Si bien se la considera como una ampliación del régimen de la seguridad social, la AUH consta de condicionantes dinerarios del comportamiento de las madres administradoras. Como el mismo documento lo indica: las madres tienen prioridad para cobrar la AUH. Por ello, en la práctica, la mayoría de las personas que reciben la transferencia son mujeres. La norma menciona que: "la mujer es uno de los pilares fundamentales en el que se apoya la familia y la sociedad, teniendo un rol fundamental en el cuidado de los hijos. Que dicha condición la hace esencial al momento de ser la receptora de los recursos otorgados por la Seguridad Social para dar cobertura a los niños, adolescentes y personas con discapacidad". [N. de la E.]

Los beneficiarios deben pertenecer a grupos familiares que se encuentren desocupados o se desempeñen en la economía informal con ingresos inferiores al salario mínimo vital y móvil (SMVM). La prestación monetaria no retributiva es de periodicidad mensual y se abona a uno solo de los padres o tutores. Para ampliar, véase el Decreto 1602/2009 (BO del 30 de octubre de 2009), "Asignaciones familiares", en http://www.ilo.org/dyn/travail/docs/1804/DECRETO\%20 1602.pdf 
Teniendo en cuenta la cantidad de menores que se ven beneficiados por esta política y los efectos positivos no solo de corto sino de largo plazo que significan las condicionalidades de salud y educación, no cabe dudas que la Asignación Universal por Hijo es uno de los programas de asistencia social más importante que se ha implementado en los últimos tiempos, con un fin claro de equidad distributiva. (Anses 2011, 5)

Por lo tanto, y teniendo en cuenta una especie de consenso entre beneficiarios e implementadores de estas transferencias sobre el dinero-capital que implican, en este artículo pregunto: ¿qué ocurre cuando uno de los beneficiarios, en vez de continuar estudiando y acumular capital humano, elige dejar la escuela y dedicarse a un empleo que le ofrece mayores ingresos que la AUH?

\section{Acumular capital humano en los márgenes}

En la ciudad de Paraná, entre el 2017 y el primer semestre de 2018, a través de un muestreo de casos seleccionados pseudoaleatoriamente, realicé una serie de encuestas, grupos focales y entrevistas en profundidad a beneficiarias de las transferencias monetarias $\mathrm{AUH}^{5}$. El objetivo principal fue determinar qué incidencia tenían distintos futuros imaginados en los consumos - reales y proyectados - de bienes y servicios, con especial énfasis en los servicios estatales de salud y educación. En conjunto con las receptoras de dichos programas, realizamos alrededor de 120 presupuestos familiares en los que se consignaron ingresos, egresos y modos de administración de las respectivas unidades domésticas. A partir de esta información, confeccioné presupuestos de hogares típicos y propuse varias categorías de receptores de las transferencias monetarias y usos comunes y diferentes de esos dineros (Dapuez et al. 2017). Uno de esos casos será analizado en este artículo. Se trata de una familia cuyo ingreso total supera la media de las familias receptoras de AUH relevadas ${ }^{6}$.

5 En el marco del proyecto de investigación orientada que dirijo (Conicet-UNER 14620140100048CO), detectamos que varios beneficiarios de la AUH, en general varones menores, intentaban abandonar o efectivamente abandonaban la escuela secundaria para trabajar en ocupaciones precarias y temporales, entre las que se destacaban las de construcción. Para más información sobre sus trayectorias educacionales, sugiero ver la tesis doctoral en ciencias sociales de Laura Raffo (2020) en la UNER, que también dirigí.

$6 \quad$ El presupuesto mensual del hogar que describo se compone de los siguientes ingresos al mes de marzo de 2020: uno altamente variable, proveniente de la pareja de Marina, de alrededor de ARS 15.000 (USD 175 aproximadamente) obtenidos por la manufactura y venta de ladrillos; un salario social complementario - para realizar tareas comunitarias administrado por el gremio 
En una segunda etapa de la investigación, a partir de agosto del 2018, analicé tanto fuentes primarias (entrevistas y encuestas con funcionarios, expertos y receptores de las transferencias monetarias) como secundarias (Censo de Población y Vivienda, informes de programas sociales de desarrollo), para emprender la escritura de distintos artículos sobre las características locales de implementación de la AUH en la provincia de Entre Ríos. En varios me he dedicado a determinar los aspectos sociodemográficos de las unidades domésticas receptoras de $\mathrm{AUH}$, tomando en cuenta principalmente los presupuestos familiares de las unidades domésticas como objeto de investigación. Por medio de técnicas de recolección de datos que combinan el método etnográfico, la creación de presupuestos y ciertas herramientas de la demografía — siguiendo los estudios de Fricke (1994), Guyer (1997) y Paolisso et al. (2002)—, he realizado una primera categorización de los hogares receptores de transferencias monetarias en la provincia de Entre Ríos. Cabe aclarar que, de cualquier manera, la principal técnica para recolectar estos datos fue la entrevista en profundidad sobre presupuestos familiares. Aunque los informantes pueden ser muy reservados acerca de sus gastos, los presupuestos de las personas de bajos ingresos son muy poco elásticos: en su mayor parte se destinan al rubro alimentación, aunque hay excepciones. En la investigación de campo también entrevisté a trabajadores de empresas de préstamos predatorios, que ofrecían dinero a altas tasas de interés para la compra de teléfonos celulares y motocicletas. Según las administradoras de AUH, solo una minoría de ellas se había involucrado en este tipo de inversiones.

local de los ladrilleros, relacionado con el Movimiento Evita- de ARS 8.500 (USD 99 aproximadamente), proveniente del Estado nacional; tres transferencias monetarias condicionadas AUH - una por discapacidad y otras dos para la protección social-, una proveniente de la tarjeta alimentaria y una del programa Hacemos Futuro para madres vulnerables. Sumados dan un parcial de ARS 25.500 (USD 298 aproximadamente) y un total de ingresos de ARS 49.000 (USD 572 aproximadamente) por mes, en el mejor de los casos. Marina vive con dos de sus hijos - un varón discapacitado de 15 años y una niña de 13 años-y su pareja —de 38 años- en una casa hecha con maderas, chapas y plástico. Su hijo mayor, Lucas, vive con su novia en una habitación o "casa" adyacente. Lucas y su novia se alimentan por lo menos una vez al día de la cocina de Marina. Esporádicamente, contribuyen con alimentos o dinero. Uno de los reclamos más persistentes de Marina es hacia Lucas, para que él provea de alimentos a su novia y se independice económicamente del hogar materno. 


\section{Dinero-capital e inversión en capital humano}

En el caso que voy a presentar, un joven de quince años entra en el mercado de trabajo como albañil temporario, con lo cual no solo pone en cuestión el objetivo explícito del programa AUH, sino también la relación con su madre. En resumen, Lucas reprueba el año, consigue trabajo informal en la construcción y no quiere volver a la escuela; así inicia una discusión con su madre sobre la responsabilidad materna y sobre los retornos económicos que de él se pueden esperar, a mediano y largo plazo, si abandona la escuela.

En este caso, las expectativas de obtener un buen trabajo una vez finalizada la escolarización tienen gran importancia en el cálculo que las personas realizan sobre sus vidas. Aunque la fase de inversión en capital humano a largo plazo ha sido desatendida en la mayor parte de la literatura sobre transferencias monetarias y economías populares, el caso aquí analizado permite entender que las madres administradoras de las transferencias pretenden que sus hijos acumulen el capital humano necesario para obtener un buen trabajo, pero siempre haciendo un cálculo entre los costos a corto plazo versus los beneficios en el largo. En el caso de Marina (ver nota a pie 6), como se mostrará más adelante, la independencia económica y alimentaria de su hijo conspira en contra de este objetivo.

En general, las madres administradoras de AUH proyectan la inversión en capital humano más allá de los procesos de educación formal, secundaria y, en muchos casos también, terciaria y universitaria. Tanto las receptoras de AUH como los beneficiarios conocen perfectamente el objetivo de la política: acumular capital humano para mejorar sus perspectivas laborales en el largo plazo. Aquí, las orientaciones económicas populares coinciden con los diseños expertos de la política de transferencia monetaria (Fiszbein et al. 2009).

No son solamente las madres quienes consideran que los fines de la transferencia monetaria se orientan principalmente a este tipo de acumulación de capital humano en sus hijos. Sectores bajos y medios de la sociedad hacen el mismo diagnóstico: una cierta educación, salud y alimentación acumulada en los hijos es la condición de posibilidad para que puedan lograr ascenso social y obtener "un buen trabajo". En Argentina, los discursos modernistas sobre la razón de ser de la escuela pública, gratuita y obligatoria y sobre la relación entre madres e hijas/os preceden y justifican la búsqueda de acumular capital humano. En suma, las transferencias monetarias de AUH y su dinero-capital refuerzan y modifican 
ideas, argumentos, debates y políticas públicas en torno a la gratuidad de la salud y la educación favorables a la inversión en capital humano.

Uno de los objetivos de este artículo es, por lo tanto, dejar en claro que, al menos entre algunos sectores pobres de la Argentina, circula un dinero-capital promovido por la AUH y que está relacionado con nociones intuitivas de capital humano. Fragmentos de varios discursos estatales sobre la utilidad económica de la educación son permanentemente recortados, apropiados y proyectados como prefiguraciones de mejores futuros posibles para los alumnos de bajos recursos. Recreando nuevas culturas del monetarismo (Guyer 2016, 75, 110), programas como la AUH sitúan a las administradoras y a los beneficiarios en una de las zonas más intensas de formación del capital, en este caso, el humano7.

Numerosos funcionarios del Estado argentino, algunos de los cuales implementan y controlan los programas de transferencias monetarias, parecerían concebir estos dineros en términos no capitalistas $^{8}$, es decir, no como una renta de un capital. ¿Por qué? Al presuponer que los dineros distribuidos por dichos programas solamente ofrecen a sus destinatarios un incremento de liquidez y nuevas posibilidades de consumo, los beneficiarios de las transferencias monetarias son imaginados y representados como receptores y administradores de un dinero simple, que no aseguraría en ningún caso un retorno de capital. Así, los programas de transferencias monetarias, se cree, funcionarían como un mecanismo compensatorio al redistribuir ingresos hacia quienes no se benefician de los circuitos económicos “capitalistas”.

Como lo sugieren Fotta y Balen (2019), el Estado nación marginaliza a través de prácticas de exclusión-inclusión (11), al mismo tiempo que marca a sus pobres como poblaciones a incluir. Pero, a pesar de que este mecanismo estatal sea

7 Una parte de la bibliografía especializada, por ejemplo, Hanlon, Barrientos y Hulmes (2010), quienes hablan de una revolución desarrollista desde el sur global —noción que también reproduce Ferguson $(2015,13)$ - , ubica en dos países latinoamericanos, el Brasil de 1995 y el México de 1997, el origen de las transferencias monetarias condicionadas. Sin embargo, estas fueron diseñadas, implementadas y rediseñadas en los Estados Unidos y Canadá durante la revolución paradigmática del monetarismo. Lena Lavinas $(2013,13)$ sitúa en un informe de 1968 del economista estadounidense Richard Zeckhauser, presentado a la RAND Corporation, la idea de asociar condiciones a la asistencia social. Esta historia de la economía se podría saldar si se leyeran con cuidado las cronologías y los análisis de las políticas de transferencias monetarias y programas de ingresos garantizados durante los años setenta. Sobre la experimentación social con estos, se puede consultar, por ejemplo, Hum y Simpson (1993).

8 El decreto presidencial de creación de la AUH entre sus considerandos reza que "no implica necesariamente el fin de la pobreza, pero inocultablemente ofrece una respuesta reparadora a una población que ha sido castigada por políticas económicas de corte neoliberal" (Decreto 1602/2009). Sin embargo, el hecho de que la AUH, como otros programas de transferencias monetarias condicionadas, haya sido diseñada por medio de un saber experto relacionado con la economía conductista y el monetarismo lleva a preguntar hasta dónde podría ser efectivo utilizar una herramienta del neoliberalimo para paliar sus propios efectos. 
analizado como lo hacen los autores citados, el proceso de reproducción capitalista no se agota en la participación del Estado como estigmatizador de poblaciones, en el sentido de marcar a los no incluidos, ni tampoco en la redistribución de los ingresos del conjunto de la sociedad. La inyección de dinero-capital en forma de transferencias monetarias para sacar de la pobreza a grandes sectores poblacionales difícilmente puede ser explicada en términos de marcación del dinero (Zelizer 1994). La domesticación de los dineros, a través de su personalización, no agota las posibilidades de inversión y retornos de un dinero-capital.

Es interesante destacar el aporte de Simone Polillo a la perspectiva de Zelizer sobre marcación del dinero, dinero industrial y capitalización generalizada del dinero. Polillo (2017) incluye la noción de Veblen de dinero industrial como mediador entre el mundo de los negocios, "un mundo aparentemente separado de las relaciones que conciernen a los hogares”, y estos últimos. Sin embargo, al sostener que "presupuestar un dinero para invertirlo es marcarlo”, Polillo $(2017,89)$, al igual que Zelizer, cae en una especie de simplificación. ¿No será que los sectores de escasos recursos presupuestan sus gastos a futuro siguiendo el modelo de las empresas y los negocios? Lo contrario sería sostener que la marcación de dineros de los sectores menos pudientes que estudia Zelizer preexiste y modela prácticas empresariales. Un caso opuesto es el que presenta Becker (1981), quien considera a los hogares en sus decisiones de inversión como complejas empresas capitalistas. En pocas palabras, invertir es mucho más explicativo que marcar.

Usos y abusos de la noción marcación de Zelizer han derivado en tantas interpretaciones como intérpretes culturalistas se han empeñado en contradecir hechos básicos como que el Estado capitalista es el principal acuñador de moneda; que los pobres tienen poco dinero y los ricos mucho; que pobreza y riqueza son relaciones básicamente económicas; y que, por más marcas que un pobre le haga a una cantidad escasa de dinero, no se puede contradecir o contrarrestar absolutamente el poder semiótico de un Estado acuñador e inversor o de un millonario que dispone de grandes sumas para contratar y comprar grandes cantidades de bienes y servicios. Y, en este caso, no se puede contrarrestar que el Estado invierta en capital humano de sus sectores más pobres.

La inversión en capital humano que realiza el Estado a través de programas de transferencias condicionadas se objetiva en la implementación de un nuevo dinero-capital. Baste aclarar ahora que las diferencias teóricas entre dinero neutral ${ }^{9}$, el que ofrece solamente una liquidez - y que termina realizándose en entenderlo como algo desacoplado de la productividad y de la producción general. El monetarismo considera que el dinero es neutral en el largo plazo, es decir, que la cantidad de 
un consumo- y el dinero-capital — que asegura retornos económicos a futuro- han sido ampliamente estudiadas desde diversas perspectivas teóricas por Marx (2000, 103-129), Friedman (1969), Keynes (1936), Lagos y Rocheteau (2008) y Monnet (2015), entre otros. En resumidas cuentas, la mayor apuesta de este artículo es recalcar que el dinero de AUH aparece también a los ojos de sus receptores como una forma particular de capital que "genera un retorno real" (Monnet 2015, 35): el que proviene de la acumulación de capital humano. Por lo tanto, el descubrimiento de un dinero-capital entre los sectores más pobres de una sociedad probaría una nueva efectividad económica de las transferencias: la de reproducir a sus beneficiarios como agentes capitalistas.

\section{Marina y Lucas: trabajo, dinero y capital humano}

Marina vive en un asentamiento de la ciudad de Paraná. Se trata de una barriada de viviendas de madera y chapas que consta de dos parcelas divididas por una avenida. El asentamiento se formó alrededor de la explotación de hornos de ladrillos, con una mayoría de migrantes del pueblo entrerriano de Santa Elena. Llegué ahí a instancias de un representante del gremio de ladrilleros. El barrio tiene una fuerte identidad política marcada por esta agrupación. Sus habitantes, más allá de que trabajen o no en la producción artesanal de ladrillos, se autodenominan ladrilleros para realizar reclamos ante políticos y gobernantes.

Marina tiene tres hijos y vive con su pareja. Lucas, su hijo mayor, ya hace dos años que se construyó una vivienda con cartones junto a la de su madre. Según me lo contó, lo hizo para cohabitar con su novia. Las dos unidades domésticas están muy cerca y muchas veces funcionan como habitaciones de un conjunto mayor, en donde circulan alimentos, préstamos de dinero y objetos, favores y otros servicios. Los presupuestos de ambas están íntimamente relacionados. La AUH de Lucas es cobrada y administrada por su madre. Lucas tiene otros ingresos esporádicos, pero regularmente le pide dinero y ayudas materiales a ella, principalmente para comida y ropa.

dinero no afecta finalmente a la economía real. Keynes y los poskeynesianos rechazan este supuesto, tanto en el largo como en el corto plazo. 
Cuando se refiere a algunos de sus problemas, en este caso, uno relacionado con nuestra investigación, Marina me explica por qué Lucas tiene que repetir el tercer año de la escuela secundaria:

También fue mi culpa... este año... porque todos los años... porque él nunca pasó libre, siempre pasaba rindiendo. Todos los años llegaba el día de la mesa de examen, yo le daba la plata, el documento, y él iba y se inscribía. Había sido que este año tenía que ir yo a inscribirlo, ¿viste? Y yo confiada... encima lo ayudé, lo preparé, le hice un resumen, por lo menos de las dos materias. Las otras las iba a sacar este año. Pero cuando llegó, no lo dejaron rendir porque tenía que ir yo a inscribirlo, así que repitió. (Marina, entrevista, diciembre de 2018)

Marina se culpa a sí misma porque su hijo ha repetido el año y ahora reflexiona sobre la posibilidad de que ese fracaso escolar haya sido la causa de que Lucas esté trabajando de albañil. Aunque ella envió a su pareja a anotarlo en otra escuela, Lucas se rehusó:

Lo anoté en la Escuela Hogar. Conseguí banco. De once de la mañana a cinco de la tarde. Porque ellos entran y tienen talleres. A mí me pareció una escuela bien... fue el Lázaro a anotarlo. Y le mostraron. Y entran a las once, hasta la una tienen taller, comen... Dicen que comen rebién. Le dan la comida, todo. Y después, de una a cinco, la escuela. ¡Y no! Se enojó. Dice: “Yo no voy a ir”. Pero mi mamá le influye mucho: “¿Cómo lo vas a mandar ahí? Esa escuela es... va a venir el otro... Como está en un barrio feo, dicen...”. No sé. Yo no conozco. No quiere ir. Y, aparte, ya está trabajando y está viendo la plata. (Marina, entrevista, diciembre de 2018)

Marina pudo haber inscrito personalmente a su hijo, en dos escuelas distintas, aunque no lo hizo. Se siente culpable de haberlo enviado a él y a su pareja a anotarlo. Cree no haber cumplido con una condicionalidad del programa. Pero esto no es lo más importante. Lo que está en juego, según ella, es el futuro de Lucas. Este futuro se complica aún más, de acuerdo con Marina, porque es posible que la novia de su hijo también pierda el año y deje la escuela. La deserción escolar de Lucas significa que Marina perderá el dinero de la Asignación Universal para la Protección Social del año que viene, pero también algo más importante: un futuro para él.

Y yo le dije al Lucas:

-Yo no voy a cobrar más la asignación si vos no vas. Está bien, no importa, lo que yo quería era que vos estudies.

Y [Lucas] me dice:

-Ahhhh... iporque cobrás! 
— ¡No! Si yo cuánto tiempo cobré la asignación familiar. Cobraba, me acuerdo... Cuando recién empecé a cobrar [eran] \$150, \$130, y yo te obligaba a ir a la escuela [lo mismo] porque no me importaba. Es más, cobraba cada tres o cuatro meses [...] porque para juntar eso...

No es por la plata... Porque si no hubiese cobrado nada, ellos iban a ir a la escuela igual. (Marina, entrevista, diciembre de 2018)

Marina disminuye el poder condicionante de la transferencia de AUH. La condición que ella cree no haber cumplido, inscribir a su hijo, es relativizada con el ejemplo del pasado: cuando ella recibía la escasa asignación familiar y enviaba "igual” que ahora a sus hijos a la escuela. Al comparar la plata de la AUH con la de la Asignación Familiar que cobraba cuando ella trabajaba, minimiza el efecto que el dinero podría tener para responsabilizarla, en este caso, de inscribir personalmente a su hijo. Ella mandaría a la escuela a Lucas reciba o no la AUH. Pero si la escuela no certifica su asistencia, el Estado automáticamente discontinúa la asignación.

Según Lucas, ella lo obliga a ir a la escuela porque quiere seguir cobrando la AUH. El dinero está condicionado a la asistencia de Lucas a la escuela, entonces, le hace pensar a él que es esa la razón más importante para que su madre lo obligue a escolarizarse. Pero, para la madre, el dinero y la escuela contribuyen a un logro mayor: que Lucas pueda acumular ciertos conocimientos, habilidades y destrezas que le permitan vivir mejor en un futuro de largo plazo.

\section{Formas del capital}

Con el surgimiento de la noción de capital humano, los conceptos de riqueza y de pobreza incorporaron habilidades y destrezas humanas que anteriormente no habían sido consideradas como objetos económicos. En particular, esta nueva forma de capital se vuelve fundamental para explicar, por ejemplo, "la rápida recuperación de posguerra de países que han sufrido destrucción severa de sus plantas y equipamientos durante la guerra” y, también, el estado perenne de subdesarrollo de otros países que solo asignan un rol prioritario a la formación de capital económico al presuponer la superabundancia de sus recursos humanos (Schultz 1961, 16). Theodore Schultz $(1961,1)$ explica que el crecimiento económico de los Estados Unidos solo puede ser deducido de las cualidades acumuladas en su población, por medio de la educación, servicios de salud, entrenamiento laboral y la migración para conseguir mejores oportunidades. 
Los trabajos de Mincer (1958, 1981), Schultz (1961), Becker ([1964] 2009) y posteriormente Sen (1997) y Goldin (2016), entre otros, no solo estudian un objeto nuevo, el capital humano; también establecen que su carencia es una causa eficiente de la pobreza ${ }^{10}$. Sin embargo, la descripción de capital humano del monetarismo depende del reconocimiento de áreas de la realidad social existentes por fuera del mercado y que antes no habían sido consideradas como fenómenos económicos.

A este proceso de descubrimiento, reconocimiento y apropiación disciplinaria de la vida cotidiana se lo ha denominado críticamente economización de la vida (Murphy 2017, a partir de Foucault [1978-1979] 2007, 262). Como tan bien lo señaló Michel Foucault ([1978-1979] 2007) hace más de cuarenta años, la misma noción de capital humano implica la descomposición del factor trabajo, noción no solo de sentido común, sino también uno de los pilares de la economía política a partir de Adam Smith, pero esta vez desde la misma perspectiva del trabajador. Al comportar el trabajo un capital (humano), "es decir una aptitud, una idoneidad" incorporada y, por otro lado, un ingreso, un salario o "un flujo de salarios" (263), la descomposición del trabajo en capital y renta induce, desde luego, a una cierta cantidad de consecuencias bastante importantes. Si, como lo señaló Foucault, el objeto capital humano fue fundamental para descomponer el concepto económico de trabajo y recomponerlo en términos neoliberales, una de las más importantes intervenciones de un Estado capitalista será solucionar las trampas de mercado por medio de transferencias monetarias.

A más de cuarenta años de este análisis, la acumulación de capital humano se transformó en el objetivo último de varias políticas. Dicha acumulación no es solamente una finalidad en sí misma ${ }^{11}$, sino también un objetivo a largo plazo, como lo refieren mis informantes, de un capitalismo que nunca termina de lograrse. Lo que se designa en términos de capital humano comienza a formar

10 Esta carencia ha sido luego relacionada con una serie de mecanismos perversos, fallas o trampas que el mercado ejecuta. Estas impiden que la capitalización intergeneracional de ciertas destrezas, habilidades y conocimientos, en definitiva, de capital humano, den su fruto (Mayer-Foulkes 2007). Mayer-Foulkes no solo analiza así lo que él denomina fallas de mercado en capital humano en México. Lo que hace es relacionar causalmente las trampas generadas a partir de la carencia de capital humano con la imposibilidad de la acumulación de múltiples capitales, y comienza circularmente por el nuevo origen de la riqueza, el capital humano. Su propuesta aboga por que el Estado solucione o, por lo menos, modere estas fallas mercantiles a través de inversiones en capital humano. Sin embargo, el concepto originario de capital humano posee una potencia explicativa, para ponerlo de alguna manera, mucho mayor a la de las trampas del mercado.

11 Para un análisis teórico, véase Dallorso (2013). 
parte del sentido común de los mismos sujetos económicos y transforma subjetividades sin las cuales no se puede pensar la vida económica activa ${ }^{12}$.

A diferencia de otros análisis que objetivan el dinero de las transferencias monetarias como piezas de dineros donados o militados (Wilkis 2013), mis informantes consideran que los dineros de la AUH deben ser capitalizados principalmente para el bienestar y la educación de los beneficiarios. Dicha inversión, según Marina, redundará en beneficios económicos a mediano y largo plazo, lo que su hijo en ningún caso contradice. La decisión de Lucas de abandonar la escuela no se basa en un diagnóstico distinto sino en entender que él no podrá terminar con esta inversión y, por lo tanto, que en su caso no será posible capitalizarla. Aunque las etnografías de procesos de inversión popular y de sus ecologías (D’Avella 2014) sean escasas, existe acuerdo sobre un ethos capitalista particular de las clases medias (Adamovsky, Visacovsky y Vargas 2014) en el que se resalta la potencialidad de las inversiones en educación, alimentación y salud para el ascenso social en la Argentina del siglo XX.

\section{Derecho al capital y obligación de la madre}

A diferencia de toda una corriente bibliográfica que considera la AUH desde la perspectiva de derechos (Abramovich 2006) o de ampliación de derechos (Mazzola 2015), mi interpretación no intenta una justificación jurídica de la actual fase del capitalismo, denominada capitalismo virtual, porque sus características son mayormente imaginarias y virtuales (Hart 2001, 313). Mi interés no es dar cuenta de la emergencia de nuevas formas jurídicas que intentan objetivar las cambiantes relaciones del capital con el trabajo en la actualidad. Sin embargo, tomo seriamente una noción proveniente del monetarismo y analizo sus diversas reinterpretaciones porque esta forma parte fundamental del dispositivo transferencias monetarias; es más, está explícitamente indicado como su objetivo principal a largo plazo. ¿Pero qué pasa cuando no se alcanzan los objetivos deseados? ¿Se

12 Según Nicolás Dallorso (2013): "La teoría del capital humano, al proponer un esquema de razonamiento que introduce en el análisis el factor tiempo, dado que una decisión intertemporal de inversión en un stock de conocimientos garantizaría un rendimiento futuro, se presenta como un adecuado conjunto de hipótesis explicativas para articular los objetivos de corto y largo plazo de las TMC y para la gestión política de las conductas y los comportamientos de los individuos en situación de pobreza" (118). En este sentido, una forma determinada de gestión de la pobreza, como también lo señala Agudo Sanchíz (2015), es que los pobres se conciban como sujetos en formación, en los que la acumulación de capital humano, una de las principales funciones de las TMC, sea parte de sus pedagogías. 
mejoran el sistema de salud o el sistema educativo, cuyas demandas han sido incentivadas por las transferencias? No. En vez de invertir en educación (mejores escuelas, mayores salarios para los docentes, etc.) y salud (mejores hospitales, mayores salarios para los profesionales de la salud, etc.) de la población, se le comunica que fue provista de más derechos —esta última opción es más barata-

Al disimular los condicionantes del comportamiento en términos de corresponsabilidades de las madres para con sus hijos, el autodenominado nuevo paradigma de ampliación de derechos ( $v$. g. Kliksberg y Novacovsky 2015; Mazzola 2015) produce una descripción de las transferencias monetarias más como un derecho que como un dinero. Desde una posición estadocéntrica, ese "derecho" del beneficiario desplaza el eje económico de análisis de un programa contra la pobreza. Se lo interpreta principalmente como formación de un nuevo tipo de ciudadanía. A ese nivel discursivo, la gubernamentalidad como "conciencia de sí del gobierno” (Foucault [1978-1979] 2007, 17) se expresa principalmente a través de funcionarios e investigadores que priorizan una forma jurídica del capitalismo (17) por sobre su manifestación económica.

En consecuencia, la acumulación de capital humano, objetivo manifiesto de las transferencias condicionadas, queda de presente solamente en un cúmulo de buenas intenciones. La evaluación de la efectividad del programa se hace más dificultosa al expresarse en términos de derechos, ya no de vacunaciones y chequeos de salud provistos, de certificados escolares y de puestos de trabajo obtenidos. La magia monetarista del Estado también es performativa: da derechos por una módica suma. Comparada con la construcción de nuevas escuelas, aggiornamiento del currículo escolar, prestación de salud de calidad, etc., es ínfima. Así, tanto el discurso de los funcionarios estatales como el de la autodenominada perspectiva de ampliación de derechos, al utilizar nociones de reconocimiento y justicia distributiva (Fraser y Honneth 2003) aplicando estos ideales a las transferencias monetarias (Ferguson 2015), desplaza el problema desde lo infraestructural hacia lo cultural. La supuesta utilidad de las transferencias es subsanar fallas del mercado sin que el Estado realice las grandes inversiones que son necesarias en salud y educación. Al enfocarse en incentivar la demanda de salud y educación, con un énfasis en los comportamientos de las madres, se presupone que el funcionamiento de los sistemas de salud y educación es óptimo o, por lo menos, bueno. ¿Por qué? Porque la teoría que le diera origen, allá por los años sesenta en Estados Unidos, así lo presuponía.

Pero, como lo sostiene Agudo Sanchíz (2015), del análisis de las intencionalidades de los discursos de las políticas no puede deducirse que los gobernados se constituyan a sí mismos como sujetos gobernables (18) ni que estas intenciones alcancen. En este caso particular y aunque algunos investigadores promuevan la 
perspectiva de ampliación de derechos o, desde el discurso oficial, tanto el presidente Mauricio Macri como la presidenta Cristina Fernández hayan coincidido en que la AUH no debe ser considerada "como un regalo, sino como un derecho" (“La buena imagen del kirchnerismo” 2015; Lewkowicz 2010), la AUH es percibida por la mayoría de sus receptores -Marina y Lucas entre ellos-como un dinero a invertir, en la ingente tarea de ganarse la vida. Si bien una cosa es lo que dice el discurso de la política y otra lo que los beneficiarios hacen con ella, estos últimos entienden bien cuál es el objetivo de la política: acumular capital humano para salir de la pobreza y efectivamente tener más derechos. Esta inversión no depende de su denominación esporádica. Mencionadas o no en términos de capital humano, según se utilice o no el lenguaje experto, la intencionalidad y la efectividad de los programas de transferencias monetarias, creados a su vez por economistas educados en Estados Unidos, deben ser primero analizadas en sede económica. Del éxito o fracaso de esta acumulación depende, luego, el verdadero alcance de las políticas y sus posteriores análisis críticos en contextos divergentes.

Las políticas de transferencias monetarias, si bien surgieron como alivios momentáneos para que se realizaran cambios estructurales en los años noventa (Levy 2008), luego prolongaron en el tiempo el objetivo manifiesto de la acumulación de capital humano en sus beneficiarios a través de diversas implementaciones. Como lo sostienen mis entrevistados y los expertos que diseñaron los programas de transferencias monetarias, el objetivo principal de la AUH, y de las transferencias monetarias condicionadas en general (Fiszbein et al. 2009), es lograr una mejoría en las potencialidades laborales de sus beneficiarios, a través de la mentada acumulación de capital humano.

\section{Ir a la escuela: ¿por el dinero de la AUH o para acumular capital?}

Las implementaciones de los programas de transferencias monetarias han afectado de distintas maneras la maternidad de las receptoras-administradoras, la pobreza y la misma naturaleza social del dinero - ver, por ejemplo, en Chile, Murray y Cabaña (2018); en México, Agudo Sanchíz (2015); y en Argentina, Dapuez et al. (2017) - El caso argentino consta aproximadamente de cuatro millones de beneficiarios que reciben ARS 1.600 (USD 40) por mes y, por lo tanto, se hacen circular unos ARS 6.400.000.000 (USD 160.000.000) por mes, a montos aproximados para fines del 2018. 
En nuestro caso, el poder del dinero de AUH se pone en comparación polémica con el dinero que Lucas recibía como albañil. El poder decisorio que le da la administración de su sueldo contrasta con el que cobra y administra Marina. En un caso, cobra y administra Lucas. En el otro, cobra y administra ella. Pero al discutir la madre con su hijo la razón de por qué él debe continuar yendo o no a la escuela y al evaluar la efectividad condicionante del dinero, también se consideraron - más allá de quién posee el dinero y lo administra- los retornos económicos que la inversión educativa en Lucas pudiera rendir en distintos escenarios futuros.

Marina arguye que esos retornos probablemente serán mayores mientras más años de escolarización curse Lucas. Pero Lucas parece decidido a sostener que es el dinero presente, contante y sonante, el que manda. Él compara cantidades, parece elegir la mayor cantidad de su sueldo como albañil ante la menor cantidad que ofrece la AUH. Lucas apuesta al dinero aquí y ahora. Su madre, a los retornos de mediano y largo plazo que el capital humano acumulado en Lucas pudiera rendir. De esta manera, Marina parece tomar en serio los lineamientos principales del programa AUH sobre la acumulación de capital humano en sus beneficiarios, básicamente porque refuerza la ideología de la educación obligatoria argentina como mecanismo para el ascenso social y económico. Sin embargo, para Lucas, el problema sigue siendo el mismo:

yo estaría trabajando si no [estuviera] viviendo con ella [la señala a la madre]. No estaría comiendo con ella, pero como voy a la escuela, no puedo hacer nada... Toda mi vida hice ladrillos y como que no conozco mucho para hacer otra cosa. (Lucas, entrevista, abril de 2019)

Una vez que vuelve a la escuela, Lucas se encuentra con el mismo escenario. La madre le pide que se mantenga económicamente a sí mismo y a su novia. Que repita el tercer año y que encuentre un trabajo. “Que vea el esfuerzo”, que sepa el "valor de mantenerse”, según Marina. Teniendo en cuenta que el logro de los objetivos de la política de $\mathrm{AUH}$ depende prioritariamente de relaciones filiales entre madres e hijas o hijos, son las madres, muchas veces escasamente escolarizadas, desocupadas o precarizadas laboralmente, mal alimentadas o víctimas de violencia de género, a quienes se las hace responsables de que sus hijos continúen o vuelvan a la escuela para terminar sus estudios.

Al repetir sus expectativas de escolarizar a Lucas, Marina nos asegura algo de lo que también ella quiere convencerse: que lo mejor para Lucas es seguir estudiando y no trabajar como albañil. Marina dice tener las mismas expectativas educacionales con respecto a Lucas que cuando recibía las asignaciones familiares, cuando ella estaba “en blanco”. Pero si así fuera, el poder condicionante 
del dinero para acumular capital humano en Lucas sería nulo. Como lo podemos ver en la entrevista a Marina, ella nos quiere dejar en claro que, mediara o no la condicionalidad del dinero de la AUH, habría mandado de cualquier manera a su hijo a la escuela.

Sin embargo, después de escuchar otras entrevistas con ella y con otras mujeres del mismo barrio, sus palabras parecen dirigidas a disminuir la potencia del refuerzo conductual que el dinero de la asignación implica o la consiguiente vergüenza a la que somete a sus receptoras su pérdida, en el caso de no cumplir las condicionalidades. Perder ese dinero mensual dejaría en falta a Marina con respecto a las otras madres del barrio, quienes sí cumplen con las exigencias. La AUH refuerza, así, el mandato moral y legal que predica que los menores no deben insertarse en el mercado inmediatamente, como vendedores de fuerza de trabajo barata, sino acumular más capital humano para que, "en el futuro”, "tengan un mejor trabajo”. Marina dice sentirse responsable de que Lucas continúe en la escuela, ya que eso le asegurará un mejor porvenir a su hijo. Lucas y su madre tuvieron al respecto muchas peleas o discusiones. Ella alega que su hijo ganaba mucho más como albañil que como beneficiario de AUH, pero que se lo gastaba en ropa para él o para su novia, sin hacerla partícipe de estos gastos. En una entrevista, ella repite el reclamo que le hiciera a su hijo: "A mí no me diste nada [de la plata ganada en la construcción]... Para eso, que estudie”.

La elección de Marina por conservar la fuente de ingreso de la AUH contra un ingreso tres o cuatro veces mayor, proporcionado por el empleo de Lucas como albañil temporario, se justifica no solamente por quién administraría los dineros. Los hechos de que le paguen "un sueldo por él”, de que "[Lucas] debe estudiar” y de que "aún es muy chico para trabajar” configuran las ideas de condicionalidad que el Estado le comunica a la madre. En este punto, es crítico decidir si estas razones de consecución de capital humano para con su hijo son atendibles o si Marina solo prioriza la administración de un dinero que proviene del Estado regularmente.

\section{Volver a la escuela o tener una vida en el horno}

Cuando Marina le dice a su hijo: "Bueno, ahí está [la plata de la asignación]... ¡Estudiá! ¡Terminá la escuela!”, ella considera que el capital humano es una condición de posibilidad para salir de la pobreza. Si hubiera sido por Lucas o por su 
cálculo de corto plazo, él habría continuado trabajando como ladrillero o albañil y ganando más dinero que lo que se percibe por la AUH. Si hubiera sido por la perspectiva estatal, Marina habría reclamado y disfrutado de su "derecho" a la AUH, así como del "derecho" de escolarizar a su hijo. Sin embargo, Marina y Lucas interpretan la finalidad de estos derechos en cuanto medios para distintos objetivos. Ambos terminan considerando que la educación de Lucas no es un fin en sí mismo, sino una inversión en su futuro.

Al contarnos cómo había comenzado a trabajar como albañil con su tío, Lucas nos recuerda que ese día su tío lo vio sentado, sin hacer nada, le dijo que “tenía que acompañarlo” y fueron a hacer unos cimientos en una obra. Para él, "no hacer nada no fue nunca una opción”. Si hubiera sido solamente por él, "no volvía” a la escuela, pero el temor a que el enojo de su madre se prolongara y el dolor que ese enojo le provocó lo motivaron a inscribirse en otra escuela para recursar el segundo año del secundario. Fue su madre entonces quien ejerció, de alguna forma, una influencia para que Lucas recursara. Al considerar que “le pagan un sueldo por él”, ella logró que Lucas dejara de trabajar como albañil. Pero también el sufrimiento propio y ajeno funcionó como orientación vital en la vida de Lucas. Cuando habla del trabajo en el horno de ladrillos o en la construcción, del trabajo de su tío o del propio, lo hace con respeto, pero también con cierta pena. Puntualmente, nos dice que el trabajo en el horno "no es trabajo para nadie”. Cuando le preguntamos por qué, respondió:

y no, no es trabajo para nadie porque tenés que tener un montón de fuerza para hacer. Después llega un tiempo en el que no querés hacer más nada. Por ejemplo, mi tío, el Turco, ya anda dolorido de tantas cosas por tanta fuerza que hace con todo esto. Eso es mucho. Tener una vida en el horno no es lindo. Mi papá quiso que estudie, pero [también me dijo]: “Si no estudiás, vas a tener que trabajar”. Mi papá nunca se preocupó por mí. (Lucas, entrevista, marzo de 2019)

En otra entrevista, Lucas dice: "Trabajando en los hornos ganaba más de lo que ganaba trabajando como albañil. Yo un día me cansé [de trabajar en la construcción] y dije: 'Yo para trabajar como negro, no trabajo más’. Mi tío se fue también” (entrevista, abril de 2019). Sus palabras muestran una suerte de aprendizaje del sufrimiento y un razonamiento. Al explicar por qué no quería recursar en la nueva escuela, adonde lo habían inscrito después de que no pudiera rendir las materias previas, explica que ahí, en la Escuela Hogar, le habían dado “un puntazo ${ }^{13}$ a un amigo”: “Siempre hay problemas en la [Escuela Hogar]”. Sin

13 Herida de poca gravedad hecha con la punta de un arma o de otro instrumento punzante. [N. de la E.] 
embargo, después de tomar la decisión de volver a la escuela dice que "a mí ahora me conocen y me respetan”. Los riesgos y padecimientos que enfrenta, en el horno de ladrillos o en las tareas de la construcción, se comparan con los de retomar la escuela. Aunque esta pueda ser peligrosa y los dineros de la AUH sean escasos en comparación con los que puede ganar trabajando, él entrevé un mejor futuro en la prolongación de su educación.

\section{Capitalismo y reservas de capital humano}

Si, como dice Foucault, una vez que es inventada la noción de capital humano la retribución salarial es traducida en términos de renta, las políticas de transferencias monetarias no solo apoyan la descomposición neoliberal del trabajo -en renta y capital humano-, sino que también implican la promesa de rentabilidad creciente del capital humano a futuro. Si se persiste en situar a los receptores y administradores de las transferencias por fuera de las sociedades capitalistas contemporáneas, como si estos estuvieran completamente libres de este mandato de acumulación de capital o de gestionar cualquier tipo de relación con un capital, las políticas de transferencias monetarias serán malentendidas.

En general y con algunas excepciones como Wilkis (2013), los receptores y administradores de transferencias monetarias son considerados agentes económicos precapitalistas. Entre otros fenómenos, también se ignora que con las transferencias monetarias el Estado intenta maximizar la capitalización de transferencias gratuitas de cuidado de madres a hijas/os. Distintos regímenes de gratuidad, entre los más importantes aquellos que regulan las transferencias de bienes y servicios entre madres-hijas/os y, mucho menos, entre padres-hijas/ os, son los que sustentan la acumulación de lo que los economistas han llamado capital humano. La provisión gratuita de cuidado filial parece ser no solamente concomitante con la de la transferencia monetaria. Esta última, como toda transferencia, está definida como un traspaso de recursos de un agente económico a otro, que no tiene como contrapartida la prestación de servicios ni la entrega de bienes (Hunt 2005, 290-301). Pero las dos transferencias, la de bienes y servicios maternales y la estatal, aunque tienen como objeto al mismo beneficiario, funcionan encabalgándose una en la otra. La relación dineraria entre Estado y madres administradoras de AUH sobredetermina y se explica si y solo si se presupone un intercambio gratuito (de bienes y servicios) que estas madres prestan a sus hijos y que el dinero de la asignación sanciona. 
Así, el dinero recibido por las madres administradoras no se vuelve, en el acto mismo de su recepción y administración, un dinero radicalmente distinto al que fue distribuido por los Estados nación. Tampoco desaparece en su gasto particular el diseño que los economistas monetaristas y conductistas de Estados Unidos, primero, y organismos internacionales como el Banco Interamericano de Desarrollo y el Banco Mundial, después, le imprimieron. En el caso mencionado de Lucas y su madre, si bien ellos no hablan de acumulación de capital humano o de capacidades, ambos reconocen que el dinero de la AUH está principalmente destinado a mejorar la trayectoria educativa de Lucas. Por lo tanto, el mandato de educar al beneficiario perdura como una de las principales fuerzas de ley que el dinero comunica. Esta nos habla de las asimetrías que existen entre dadores del dinero y sus receptores y nos conduce de estudiar la mera recepción y gasto del dinero de la AUH hacia la investigación de una inversión en capital humano. Para ponerlo de una manera más simple, el imperativo de acumulación de capital humano debe ser considerado inherente al dinero de la AUH, aunque las madres receptoras y administradoras desconozcan las teorías y lenguajes disciplinarios por medio de los cuales las transferencias han sido diseñadas. La inversión estatal en el capital humano de la población más pobre incrementa el poder de compra en sus administradoras y las sujeta a más obligaciones en la formación de ciudadanos aptos para un capitalismo que no termina de ser definido. Sujeción que ellas ya experimentaban como mandato moral antes de la percepción de la asignación.

\section{Conclusiones}

James Ferguson (2015) se preguntaba si las transferencias monetarias ayudan a insertar a las personas en el capitalismo, después de — supuestamente- "haber demostrado que tales políticas no destruyen o erosionan la socialidad o la mutualidad" entre los pobres (128). Sobre el caso sudafricano sostiene que cualquier posibilidad de habitar fuera del capitalismo y de la economía dineraria (cash economy) se extinguió en el siglo XIX. Y si bien critica los programas de transferencias monetarias porque parecen tomar al "sistema capitalista" como dado y concentrarse en mejorarlo (128), en ninguna parte de su libro nos describe los nuevos dineros que emergen de las transferencias monetarias. Aunque este autor sostenga que las transferencias monetarias impulsan nuevas economías de distribución en el sur global — siguiendo en esto a Hanlon, Barrientos y Hulme (2010)_ 
y promuevan condiciones más equitativas para el surgimiento de diversas formas de vida, al mismo tiempo que advierte las amenazas de nuevas formas de abyección y de sujeción estatal, no repara en el fin último de estas transferencias: la acumulación de capital humano en sus receptores (Fiszbein et al. 2009) y la creación de un nuevo dinero-capital. Asimismo, otras investigaciones sobre transferencias monetarias han ignorado sintomáticamente las diversas formaciones y acumulaciones de capital que deberían producir. Al presuponer que las transferencias son recibidas en sectores marginales al capital, han asimilado estos últimos a las culturas precapitalistas. Otras áreas de estudios se han dedicado principalmente a estudiar las dimensiones abstractas y globales del capital humano (Downey y Fisher 2006; Kalb 2013; Murphy 2017; Streeck 2016), pero sin incorporar el estudio de las transferencias monetarias ni la creación de nuevos dineros.

Al no haber sido estudiada la formación popular de capitales, no solo se ignora un comportamiento típico de madres que invierten en el capital humano de sus hijos, sino también la producción de un nuevo dinero-capital. Aunque las receptoras-administradoras de la AUH pueden, por supuesto, renunciar a las transferencias monetarias voluntariamente, negarse a recibirlas o incurrir en faltas que impliquen para ellas y, de un año al otro, para sus hijos la discontinuidad del programa, no pueden sin embargo redefinir unilateralmente la naturaleza del capital, del dinero y del rol materno que el Estado y los organismos internacionales han previsto para ellas. Al investigar la formación de capital humano en un barrio urbano-marginal y detectar evidencias empíricas que demuestran que los beneficiarios de AUH saben qué es el capital humano, aunque evalúen sus posibilidades de acumulación de manera diferente a los economistas y los burócratas responsables de la implementación de la política, intenté contribuir al análisis de la formación desde abajo de una antropología del capital.

Los beneficiarios de políticas públicas de transferencias monetarias son los principales intérpretes de estas: ellos son los primeros y más importantes analistas de lo que es el capital humano hoy en día. Como lo muestran varias etnografías (Agudo 2015; Dapuez 2013; Murray y Cabaña 2018), la administración de la pobreza y de la precariedad ha sido altamente efectiva por medio de transferencias monetarias. Al tiempo que se controlaron situaciones sociales volátiles por medio de la distribución regular de pequeñas cantidades de dinero, la misma noción de capital cambiaba mientras el capital se extendía a nuevas áreas del planeta. En este sentido, una de las consecuencias más importantes de la implementación de políticas de transferencias monetarias es una cierta humanización del capital. Esta implica un fenómeno ambiguo. Uno de los factores de reproducción neocapitalista parece ser, de ahora en más y crecientemente, un conjunto de habilidades y conocimientos incorporados en las poblaciones 
humanas. Esta empresa global de acumulación de capital humano, por otro lado, implica que grandes sectores poblacionales sean considerados como reservorios para la producción del futuro.

Por lo tanto, la forma jurídica que toma el Estado capitalista con respecto a las transferencias monetarias u otras medidas similares de intervención, en tanto y en cuanto buscan principalmente capitalizar a sus ciudadanos, no puede ser analizada aisladamente de las necesidades prácticas, concretas y situadas del capital y de su formación. Al modificarse radicalmente el capital y su formación, al cambiar el régimen capitalista de una fase industrial a una fase de conocimiento e información, el ejército industrial de reserva parece dejar lugar a un nuevo reservorio poblacional de capital humano.

Personas como Lucas y Marina reinterpretan la acumulación de un cierto capital humano día a día. El capital humano es una de las acumulaciones más urgentes para quienes, precarizados y carentes de otras formas de capital, luchan por sobrevivir. La antropología debería investigar las variadas formas en las que el capital emerge en la vida cotidiana de la gente. En pocas palabras, habrá de surgir una antropología del capital que dé cuenta de las diversas capitalizaciones que ocurren cotidianamente. Las que un fenómeno global como el de las transferencias condicionadas produce nos plantean la necesidad de una disciplina que sea más precisa, puntual y crítica en el estudio de las formas de vida emergentes entre las políticas del Estado capitalista, sus regímenes de gratuidad capitalista, sus apropiaciones creativas, los mercados y los hogares. Si bien una antropología del capital también tendría que dar cuenta de otras modulaciones de este (Rajan 2006, 2012), la creación de una nueva subdisciplina que investigue el capital humano, su ideación, creación y reproducción como objeto principal en las sociedades capitalistas, se vuelve imprescindible para comenzar a responder a preguntas más generales sobre la naturaleza misma del capital en estos tiempos.

\section{Referencias}

Abramovich, Víctor. 2006. "Una aproximación al enfoque de derechos en las estrategias y políticas de desarrollo”. Revista de la Cepal 88: 35-50. https://repositorio.cepal.org/handle/11362/11102

Adamovsky, Ezequiel, Sergio Eduardo Visacovsky y Patricia Vargas, comps. 2014. Clases medias: nuevos enfoques desde la sociología, la historia y la antropología. Buenos Aires: Ariel. 
Agudo Sanchíz, Alejandro. 2015. Una etnografía de la administración de la pobreza. Ciudad de México: Iberoamericana.

Anses (Administración Nacional de la Seguridad Social)/Observatorio de la Seguridad Social. 2011. Asignación Universal por Hijo para Protección Social: una política de inclusión para los más vulnerables. Anses/Observatorio de la Seguridad Social, Argentina. http:// observatorio.anses.gob.ar/archivos/documentos/AUH\%20para\%20Protecci\%C3\%B3n\%20 Social.pdf

Argentina, Ministerio de Economía, Hacienda y Finanzas, Gobierno de Entre Ríos, Dirección General de Estadísticas y Censos. Ministerio de Economía, Hacienda y Finanzas. Gobierno de Entre Ríos. 2018. Tabulados básicos. Aglomerado Gran Paraná. Encuesta Permanente de Hogares. 3er Trimestre de 2018. Paraná: Dirección General de Estadísticas y Censos. https://www.entrerios.gov.ar/dgec/trabajoeingresos/

Becker, Gary S. (1964) 2009. Human Capital: A Theoretical and Empirical Analysis, with Special Reference to Education. Chicago, IL: University of Chicago Press.

-.1981. Treatise on the Family. Cambridge MA: Harvard University Press.

"La buena imagen del Kirchnerismo motiva el abrupto cambio de discurso en Mauricio Macri”. 2015. Eldiario24.com, 22 de julio. http://m.eldiario24.com/nota/356717/la-buenaimagen-del-kirchne-rismo-motiva-el-abrupto-cambio-de-discuro-en-mauricio-macri.html

Dallorso, Nicolás Santiago. 2013. "La teoría del capital humano en la visión del Banco Mundial sobre las Transferencias Monetarias Condicionadas”. Estudios Sociológicos 31 (91): 113-139. https://estudiossociologicos.colmex.mx/index.php/es/article/view/120/120

Dapuez, Andrés. 2013. "Promissory Prestations: A Yucatec Village between Ritual Exchange and Development Cash Transfer”. Tesis de Doctorado en Antropología Sociocultural, Departamento de Antropología, Johns Hopkins University, Baltimore, MD.

Dapuez, Andrés, María Laura Raffo, María Kendziur y Juan Carlos Sabogal. 2017. "De la interface monetaria madre-Estado al ombligo del dinero. Administración doméstica de la 'Asignación Universal por Hijo para Protección Social' en Paraná, Argentina”. Iberoamericana. Nordic Journal of Latin American and Caribbean Studies 46 (1): 65-76. http:// doi.org/10.16993/iberoamericana.107

D’Avella, Nicholas. 2014. "Ecologies of Investment: Crisis Histories and Brick Futures in Argentina”. Cultural Anthropology 29 (1): 173-199. https://doi.org/10.14506/ca29.1.10

Downey, Greg y Melissa Fisher. 2006. "Introduction: The Anthropology of Capital and the Frontiers of Ethnography”. En Frontiers of Capital: Ethnographic Reflections on the New Economy, editado por Melissa S. Fisher y Greg Downey, 1-30. Durham, NC: Duke University Press.

Ferguson, James. 2015. Give a Man a Fish: Reflections on the New Politics of Distribution. Durham, NC: Duke University Press.

Fiszbein, Ariel, Norbert Schady, Francisco Ferreira, Margaret Grosh, Niall Keleher, Pedro Olinto y Emmanuel Skoufias. 2009. Conditional Cash Transfers: Reducing Present and Future Poverty (English). A World Bank Policy Research Report. Washington D. C.: World Bank. http://documents.worldbank.org/curated/en/914561468314712643/Conditional-cashtransfers-reducing-present-and-future-poverty 
Fotta, Martin y Maria Elisa Balen. 2019. "Introduction: Rearticulations of Rural Livers through Conditional Cash Transfers”. En Money from the Government in Latin America: Conditional Cash Transfer Programs and Rural Lives, editado por Maria Elisa Balen y Martin Fotta, 3-30. Londres: Routledge.

Foucault, Michel. (1978-1979) 2007. Nacimiento de la biopolítica: curso en el Collège de France (1978-1979). Buenos Aires: Fondo de Cultura Económica.

Fraser, Nancy y Axel Honneth. 2003. Redistribution or Recognition?: A Political-Philosophical Exchange. Londres: Verso.

Fricke, Thomas. 1994. Himalayan Households: Tamang Demography and Domestic Processes. Nueva York: Columbia University Press.

Friedman, Milton. 1969. The Optimum Quantity of Money, and Other Essays. Londres: Macmillan.

Goldin, Claudia. 2016. “Human Capital”. En Handbook of Cliometrics, editado por Claude Diebolt y Michael Haupert, 55-86. Berlín: Springer.

Guyer, Jane I. 1997. "Endowments and Assets: The Anthropology of Wealth and the Economics of Intrahousehold Allocation”. En Intrahousehold Resource Allocation in Developing Countries: Models, Methods, and Policy, editado por Lawrence Haddad, John Hoddinott y Harold Alderman, 112-125. Washington D. C.: IFPRI; The Johns Hopkins University Press.

-. 2016. Legacies, Logics, Logistics: Essays in the Anthropology of the Platform Economy. Chicago, IL: University of Chicago Press.

Hanlon, Joseph, Armando Barrientos y David Hulme. 2010. Just Give Money to the Poor: The Development Revolution from the Global South. Sterling: Kumarian Press.

Hart, Keith. 2001. The Memory Bank: Money in an Unequal World. Knutsford: Texere.

Hum, Derek y Wayne Simpson. 1993. "Economic Response to a Guaranteed Annual Income: Experience from Canada and the United States”. Journal of Labor Economics 11 (1): 263-296. https://www.journals.uchicago.edu/doi/pdf/10.1086/298335

Hunt, Robert. 2005. “One Way Economic Transfers”. En A Handbook of Economic Anthropology, editado por James G. Carrier, 290-301. Cheltenham: Edwar Elgar.

Indec (Instituto Nacional de Estadística y Censos de la República Argentina). 2010. Censo Nacional de Población, Hogares y Viviendas 2010. https://www.indec.gob.ar/indec/web/ Nivel4-CensoNacional-999-999-Censo-2010

Kalb, Don. 2013. "Financialization and the Capitalist Moment: Marx versus Weber in the Anthropology of Global Systems”. American Ethnologist 40: 258-266. https://doi.org/10.1111/ amet.12018

Keynes, John Maynard. 1936. The General Theory of Employment, Interest and Money. Cambridge: Macmillan; Cambridge University Press.

Kliksberg, Bernardo e Irene Novacovsky. 2015. El gran desafío: romper la trampa de la desigualdad desde la infancia. Aprendizajes de la Asignación Universal por Hijo. Buenos Aires: Biblos. 
Lagos, Ricardo y Guillaume Rocheteau. 2008. "Money and Capital as Competing Media of Exchange”. Journal of Economic Theory 142 (1): 247-258. https://doi.org/10.1016/j. jet.2006.07.005

Lavinas, Lena. 2013. “21th Century Welfare”. New Left Review 84: 5-40. https://newleftreview.org/issues/ii84/articles/lena-lavinas-21st-century-welfare

Levy, Santiago. 2008. Good Intentions, Bad Outcomes: Social Policy, Informality, and Economic Growth in Mexico. Washington D. C.: Brookings Institution Press.

Lewkowicz, Javier. 2010. "La asignación llegó a los hijos de los temporarios”. Página 12, 2 de diciembre. https://www.pagina12.com.ar/diario/elpais/1-157916-2010-12-02.html

Marx, Karl. (1867) 2000. El capital: crítica de la economía política. Vol. 1. Ciudad de México: Fondo de Cultura Económica.

Mayer-Foulkes, David. 2007. "Fallas de mercado en capital humano. La trampa intergeneracional de la pobreza en México”. El Trimestre Económico 74 (295): 543-614.

Mazzola, Roxana. 2015. Nuevo paradigma: la Asignación Universal por Hijo en la Argentina. Buenos Aires: Prometeo.

Mincer Jacob. 1958. "Investment in Human Capital and Personal Income Distribution”. Journal of Political Economy 66 (4): 281-302. https://www.jstor.org/stable/1827422

-. 1981. "Human Capital and Economic Growth". NBER Working Paper Series, documento de trabajo 803, National Bureau of Economic Research, Cambridge. https://www.nber.org/ papers/w0803.pdf

Monnet, Éric. 2015. "Money and Capital. The Contributions of Capital in the Twenty-First Century to Monetary History and Theory”. Annales 70 (1): 33-44. https://doi.org/10.1017/ S2398568200000935

Murphy, Michelle. 2017. The Economization of Life. Durham, NC: Duke University Press.

Murray, Marjorie y Gabriela Cabaña. 2018. “Beyond Cash, Beyond Conditional: Ingreso Ético Familiar and the Senses of Poverty in a Group of Mapuche Women”. En Money from the Government in Latin America Conditional Cash Transfer Programs and Rural Lives, editado por Maria Elisa Balen y Martin Fotta, 162-177. Abingdon, UK: Routledge.

OIT (Organización Internacional del Trabajo). 2018. Women and Men in the Informal Economy: A Statistical Picture. Ginebra: ILO.

Paolisso, Michael, Kelly Hallman, Lawrence Haddad y Shibesh Regmi. 2002. "Does Cash Crop Adoption Detract from Child Care Provision? Evidence from Rural Nepal”. Economic Development and Cultural Change 50 (2): 313-327. https://doi.org/10.1086/322881

Polillo, Simone. 2017. "From Industrial Money to Generalized Capitalization". En Money Talks: Explaining How Money Really Works, editado por Nina Bandelj, Frederick Wherry y Viviana Zelizer, 89-104. Princeton: Princeton University Press.

Raffo, Laura. 2020. "Un bien futuro. Expectativas educacionales de las administradoras de la Asignación Universal por Hijo para la Protección Social, durante el periodo 2015-2018 en la ciudad de Paraná (Entre Ríos)”. Tesis de Doctorado en Ciencias Sociales, UNER. 
Rajan, Kaushik Sunder. 2006. Biocapital: The Constitution of Post-Genomic Life. Durham, NC: Duke University Press.

-. 2012. Lively Capital: Biotechnologies, Ethics and Governance in Global Markets. Durham, NC: Duke University Press.

Schultz, Theodore. 1961. "Investment in Human Capital”. American Economy Review 51: 1-17. https://www.jstor.org/stable/1818907

Sen, Amartya. 1997. "Human Capital and Human Capability". World Development 25 (12): 1959-1961. https://doi.org/10.1016/S0305-750X(97)10014-6

Standing, Guy. 2011. The Precariat: The New Dangerous Class. Londres: Bloomsbury.

Streeck, Wolfgang. 2016. How Capitalism End? Londres: Verso.

Wilkis, Ariel. 2013. Las sospechas del dinero: moral y economía en la vida popular. Buenos Aires: Paidós.

Zelizer, Viviana. 1994. The Social Meaning of Money: Pin Money, Paychecks, and Other Currencies. Nueva York: Basic Books. 